\title{
Declaración de posición para la atención de trauma durante la pandemia de COVID-19
}

\author{
Position statement for trauma care during the COVID-19 pandemic \\ Luis Gerardo Padilla Rojas, ${ }^{\star}$ Roberto Enrique López Cervantes, ${ }^{\ddagger}$ \\ Leonardo López Almejo, § Michael Dittmar Johnson"
}

\author{
* Cirujano Ortopedista y Traumatólogo. Hospital Puerta de Hierro Andares Zapopan, \\ Tesorero de FEMECOT, Coordinador del Módulo de Trauma FEMECOT. \\ ₹ Cirujano Ortopedista y Traumatólogo, Ortopedista en CFO (Clínica de Fracturas por Osteoporosis) \\ Sanatorio San Francisco de Guadalajara, Jalisco, México, Coordinador del FCMX \\ (Registro Mexicano de Fractura de Cadera FEMECOT). \\ $\S$ Cirujano Ortopedista y Traumatólogo, Hospital Star Aguascalientes, Ags. México. \\ Vicepresidente de FEMECOT. Coordinador del Comité Científico de FEMECOT. \\ " Cirujano Ortopedista y Traumatólogo, Hospital Puerta de Hierro Andares \\ Zapopan, Coordinador del Módulo de Columna FEMECOT.
}

FEMECOT (Federación Mexicana de Colegios de Ortopedia y Traumatología).

Correspondencia: Dr. Luis Gerardo Padilla Rojas. Avenida Empresarios No. 150-903,

Col. Puerta de Hierro, Zapopan, Jalisco, C.P. 45116. Correo electrónico: lupadilla@gmail.com

\section{RESUMEN}

Introducción: A primera vista, la epidemia de COVID-19 y el campo de la Ortopedia y Traumatología parece ser dispar. Si bien los ortopedistas no son considerados personal de primera línea en el ataque contra la pandemia, el papel del cirujano como parte del ecosistema del cuidado de la salud es crucial. Nuestra especialidad, por su extraordinaria amplitud, incide en todas las edades y en todas las magnitudes, afectando directamente la funcionalidad y la calidad de vida del paciente, por lo cual se realiza postura para la atención del Trauma con el fin de mejorar la seguridad del médico y del paciente. Resultados: Una guía esencial del manejo de nuestros pacientes con trauma musculoesquelético podría enfocarse en tres puntos. Protección: el contar con el equipo de protección

\section{ABSTRACT}

Introduction: At first glance, the COVID-19 epidemic and the field of Orthopedics and Traumatology appear to be disparate. Although orthopedists are not considered front-line personnel in the attack on the pandemic, the role of the surgeon as part of the health care ecosystem is crucial. Our specialty, due to its extraordinary scope, affects all ages and in all magnitudes directly affecting the functionality and quality of life of the patient. Therefore, a posture for trauma care is to be executed in order to improve the safety of the doctor and patient. Results: An essential guide to the management of our patients with musculoskeletal trauma could focus on 3 points. Protection: having personal protective equipment (PPE) according to the risk of each case 
personal (EPP) acorde al riesgo de cada caso y el tipo de contacto. Evitar contagio y/o diseminación: contacto con el paciente, atención de urgencias y programación de cirugía electiva o diferible. Restringir: visitas médicas, visitantes y familiares durante hospitalización y atención directa, flujo de pacientes y conglomeraciones. Uso de medios electrónicos para el seguimiento médico. Conclusiones: La literatura actual nos marca lineamientos claros sobre nuestro actuar ante este tipo de pandemia para evitar más contagios y como salvaguardo legal, aunque su nivel de evidencia es bajo y se ignoran los problemas o costos que esto pudiera generar en el futuro. Se debe de salvaguardar la vida sobre la función. Debemos evitar cometer errores que en otros países se hicieron al menospreciar este brote de COVID-19.

Palabras clave: COVID-19, pandemia, traumatología, protección, cirugía, guías nacionales. and the type of contact. Avoid contagion and/or dissemination: contact with the patient, emergency care and scheduling of elective or deferrable surgery. Restrict: medical visits, visitors and relatives during hospitalization and direct care, patient flow and conglomerations. Use of electronic means for medical monitoring. Conclusions: The current literature sets clear guidelines for our actions in the face of this type of pandemic to avoid more infections and as a legal safeguard, although its level of evidence is low and the problems or costs that this may generate in the future are ignored. Life must be safeguarded over function. We must avoid making the mistakes that other countries made of underestimating the COVID-19 outbreak.

Keywords: COVID-19, pandemic, traumatology, protection, surgery, national guides.

\section{INTRODUCCIÓN}

Definición: en diciembre de 2019 una nueva causa de neumonía fue reconocida en Wuhan, China, el COVID-19 (SARS[severe acute respiratory syndrome]-CoV-2). ${ }^{1}$

Enumeración: este brote fue declarado emergencia de salud pública internacional por la OMS (Organización Mundial de la Salud) el 30 de enero de 2020 y posteriormente como pandemia el 11 de marzo de $2020 .^{2}$ Este brote alcanzó a México el 28 de febrero de 2020, reportándose para el 29 de febrero los primeros dos $\operatorname{casos}^{3}$ e incrementándose exponencialmente, contabilizando 3,181 casos y 174 muertes para el 08 de abril de $2020 .^{4}$

Justificación: si bien los ortopedistas y traumatólogos no son considerados personal de primera línea en el ataque contra la pandemia, el papel del Cirujano Traumatólogo y Ortopédico en el control de este brote, como parte del ecosistema del cuidado de la salud, tiene un rol crucial que jugar para frenar esta pandemia viral. ${ }^{5}$

Epidemiología: en México el número de casos ha ido aumentando exponencialmente debido a la alta transmisión del agente: por contacto con fómites contaminados, por aerosoles en el ambiente, por contacto directo con el paciente o sus secreciones. ${ }^{6}$ La mortalidad del virus en México hasta el momento es de $9.7 \%,{ }^{4}$ con mayor incidencia en pacientes mayores de 60 años o acompañado de comorbilidades como diabetes, obesidad o patologías pulmonares. ${ }^{6}$

Posturas: a nivel mundial, los gobiernos han decretado posturas de aislamiento en casa de la sociedad con la finalidad de disminuir el número de conta- 
gios en un corto periodo de tiempo. Así como especialidades médicas han dado algunas recomendaciones al respecto, difiriendo cirugías electivas y pautando las atenciones de los pacientes con alguna urgencia médica o quirúrgica. ${ }^{7}$

Recomendaciones: las recomendaciones actuales internacionales vienen de los países donde el brote inició antes que en México, como lo son Inglaterra, ${ }^{7}$ Indonesia, ${ }^{8}$ Portugal, ${ }^{9}$ China ${ }^{10}$ y Estados Unidos. ${ }^{11,12}$ En donde se han tomado varias medidas como la suspensión de consultas y cirugías electivas, clasificación de los pacientes y las urgencias. Uso mayor de los tratamientos conservadores frente al quirúrgico, así como especificaciones del equipo de protección para el personal. ${ }^{13}$

Objetivo de la declaración de posición: el objetivo de esta declaración es lograr tener una postura clara de la práctica de la traumatología en el país, que funja como instrumento de guía nacional, así como de marco legal de la Ortopedia en las contingencias futuras y en la actual.

\section{MATERIAL Y MÉTODOS}

Se realizó una revisión de alcance del tema de la pandemia de COVID-19 y la práctica médica de la Ortopedia y Traumatología.

La revisión de alcance de la literatura se basó en la metodología establecida por Colquhoun. ${ }^{14}$

No existe actualmente ningún instrumento de valuación para las revisiones de alcance o Scoping Review. ${ }^{15}$

La revisión por pares se realizó por los investigadores Luis Gerardo Padilla Rojas, Roberto Enrique López Cervantes, Leonardo López Almejo y Michael Dittmar Johnson. Se realizó la búsqueda en el idioma español e inglés de los términos "COVID-19 and orthopedics/trauma", "Coronavirus in trauma surgery», "Surgeon protection COVID-19», «Recomendations for emergency health care» en las bases de datos: «PubMed», «Ovid», "Sciencedirect» y «Cochrane».

Finalmente, se realizó una descripción del tratamiento basado en la literatura revisada, plasmado de manera narrativa para un adecuado entendimiento del mismo, dividido en subtemas para abordar de una manera sistemática cada uno de los problemas a los que nos enfrentamos.

\section{RESULTADOS}

Estudios recientes han demostrado que el SARS-CoV-2 puede trasmitirse por aerosol, fómites y contacto directo ${ }^{16}$ que se ha visto que ocurre al realizar incisiones, utilización de electrocauterio, uso de herramientas de alto poder, utilizando aspiradores o por el mismo aire acondicionado del quirófano. Como se ha demostrado, el SARS-CoV-2 ha sido detectado en plasma y linfocitos, incrementando la preocupación de diseminación viral en transfusiones sanguíneas y procedimientos quirúrgicos en los que el virus puede ser aerolizado fuera del sistema respiratorio. ${ }^{16}$ 
Idóneamente todo paciente con fractura que requiere cirugía debería ser sometido a pruebas para detectar SARS-CoV-2 de manera preoperatoria, en caso de ser positivo, considerar el manejo conservador o diferir la cirugía hasta que el paciente sea caso inactivo. Sin embargo, hay pacientes que deben ser intervenidos y minimizar la morbilidad del paciente y minimizar la diseminación.

Debe considerarse hacer una separación de quirófanos para pacientes COVID-19 positivos o no conocidos y quirófanos para pacientes COVID-19 negativos o no sospechosos.

El personal en quirófano debe reducirse al mínimo, sólo residentes esenciales, no representantes comerciales o proveedores.

De manera idónea debe considerarse una sala independiente para intubación, en la cual no esté presente el resto del equipo quirúrgico. Considerar bloqueos regionales con el paciente utilizando el respirador N95. El quirófano ideal para los pacientes positivos o desconocidos sería con presión negativa de aire, a pesar que esto incrementa la posibilidad de infección. ${ }^{16}$

Generalmente el cierre de piel lo realizamos con suturas no absorbibles o grapas; sin embargo, se debe utilizar el cierre subdérmico con sutura absorbible para evitar las visitas a retiros de puntos.

Una guía esencial del manejo de nuestros pacientes traumatizados podría enfocarse en tres puntos: ${ }^{7}$

- Protección: el equipo de protección personal (EPP).

- Evitar contagio y/o diseminación: contacto de paciente, atención de urgencias y programación de cirugía.

- Restringir: visitas, visitantes, visitas médicas, flujo de pacientes.

\section{PROTECCIÓN}

Cuando se realiza un cuidado escrupuloso y el apropiado uso de EPP, la infección en los trabajadores de la salud es increíblemente bajo.

La utilización correcta de los mismos en Áreas de Urgencias (Figura 1), quirófano (Figura 2) y hospitalización debe ser apegada a los procedimientos descritos ampliamente. Hacemos sólo referencia a sugerencias de cuándo utilizarlos en la Figura 1.

\section{Evitar contagios y/o diseminación}

En el hospital se debe suspender toda actividad académica en forma presencial, realizar videoconferencias académicas y continuar el programa académico de enseñanza en línea.

Evitar o restringir el tiempo de contacto, esto se puede hacer mediante telemedicina o contactar al paciente vía remota para el seguimiento postoperatorio, disipar dudas o en lo posible sugerencias de manejo. ${ }^{17,18}$

La utilización de órtesis o férulas en lugar de yesos o inmovilizaciones circulares, reduce el número de visitas al hospital, así como conglomerar las salas de espera. 
Bajo un análisis de cada caso, hay cierta aceptación en estos momentos en la que podría ser mejor aceptar un resultado subóptimo y evitar hospitalización si existe una adecuada solución ulterior, por ejemplo, manejar conservadoramente una fractura de tobillo que probablemente lleve a una artrosis, pero disminuye el riesgo de infección por COVID-19.,8

Como cirujanos debemos dar una mayor importancia a las medidas en el preoperatorio, transoperatorio y postoperatorio de los pacientes y del personal a nuestro cargo para minimizar los riesgos de propagación hospitalaria. Esto significa programar pacientes y que se ingresen el mismo día de la cirugía.

Contactar a todos los pacientes un día previo a la cirugía y verificar si presentan algún síntoma respiratorio, o si en los 14 días previos han viajado o tenido contacto con alguna persona confirmada o sospechosa de infección por COVID-19. ${ }^{9}$

A su ingreso al hospital, verificar temperatura corporal y verificar de nuevo factores de riesgo.

Procedimientos no urgentes en pacientes mayores de 60 años de edad, con comorbilidades preexistentes, diabetes, enfermedad pulmonar, enfermedad cardiovascular, cáncer e inmunocomprometidos deben ser diferidos hasta un tiempo oportuno..$^{11,12}$

1) Casos quirúrgicos, estratificación de tiempos.

2) Emergencia $<1$ hora.

a) Ponen en riego la vida.

b) Choque hemorrágico.

c) Lesión vascular aguda.

d) Síndrome compartimental.

e) Fascitis necrosante.

3) Urgencia $<24$ horas.

a) Artritis séptica.

b) Fractura expuesta.

c) Fractura sangrante de pelvis.

d) Fractura diafisaria de fémur y/o cadera.

e) Lesión de nervio aguda/lesión de cordón medular.

f) Infección quirúrgica.

4) Urgencia electiva $<2$ segundos.

a) Colgajos, injertos, cierres de heridas.

b) Fracturas abrigadas.

c) Fracturas de columna/de acetábulo.

5) Electiva (esencial) uno a tres meses. ${ }^{12}$

a) Reconstrucción.

6) Electiva (discrecional) $>3$ minutos.

a) Reemplazo articular.

b) Cirugía deportiva. ${ }^{11}$

c) Si el procedimiento quirúrgico es necesario en un paciente sospechoso o confirmado COVID-19. 
Los procedimientos quirúrgicos ortopédicos utilizando sierras, taladros y aspiradores son generadores de aerosol, por lo que los quirófanos con presión negativa son de gran ayuda, con intercambio de aire frecuente y accesos separados. ${ }^{13}$ Asimismo, se debe utilizar el equipamiento de protección sugerido y establecer el límite de diseminación en el lugar. Dividiendo el área en cinco zonas distintas. ${ }^{9}$ Así como antesalas para colocarse y desechar el equipo de protección, incluso acondicionarlo a un lado de las salas de quirófano.

\section{Minimizar entrada y salida de quirófano}

Transoperatoriamente el equipo de protección personal (EPP), deben ser utilizadas según ciertas recomendaciones (Figura 2).

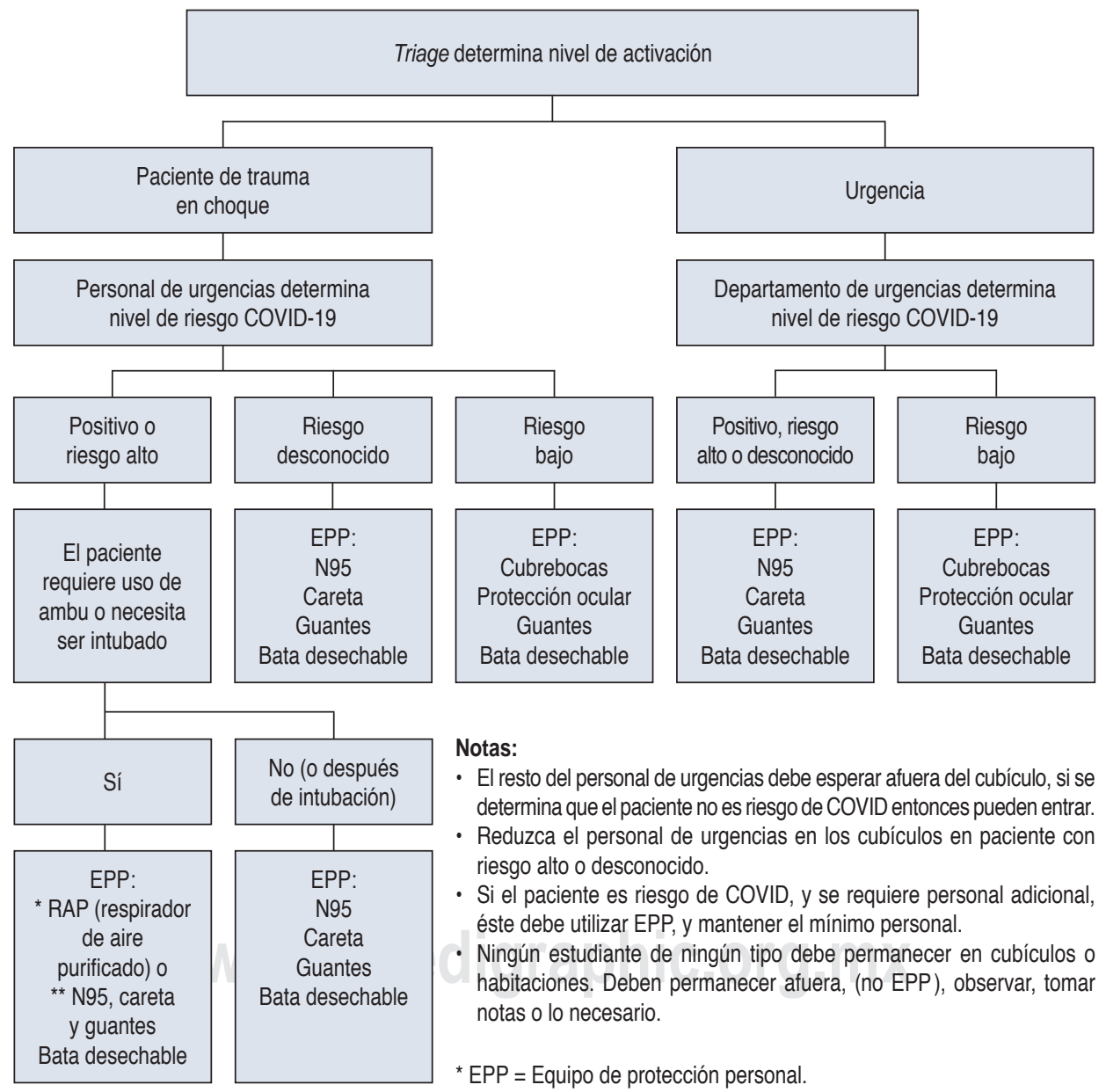

Figura 1: Nivel de protección en urgencias según el nivel de riesgo. 


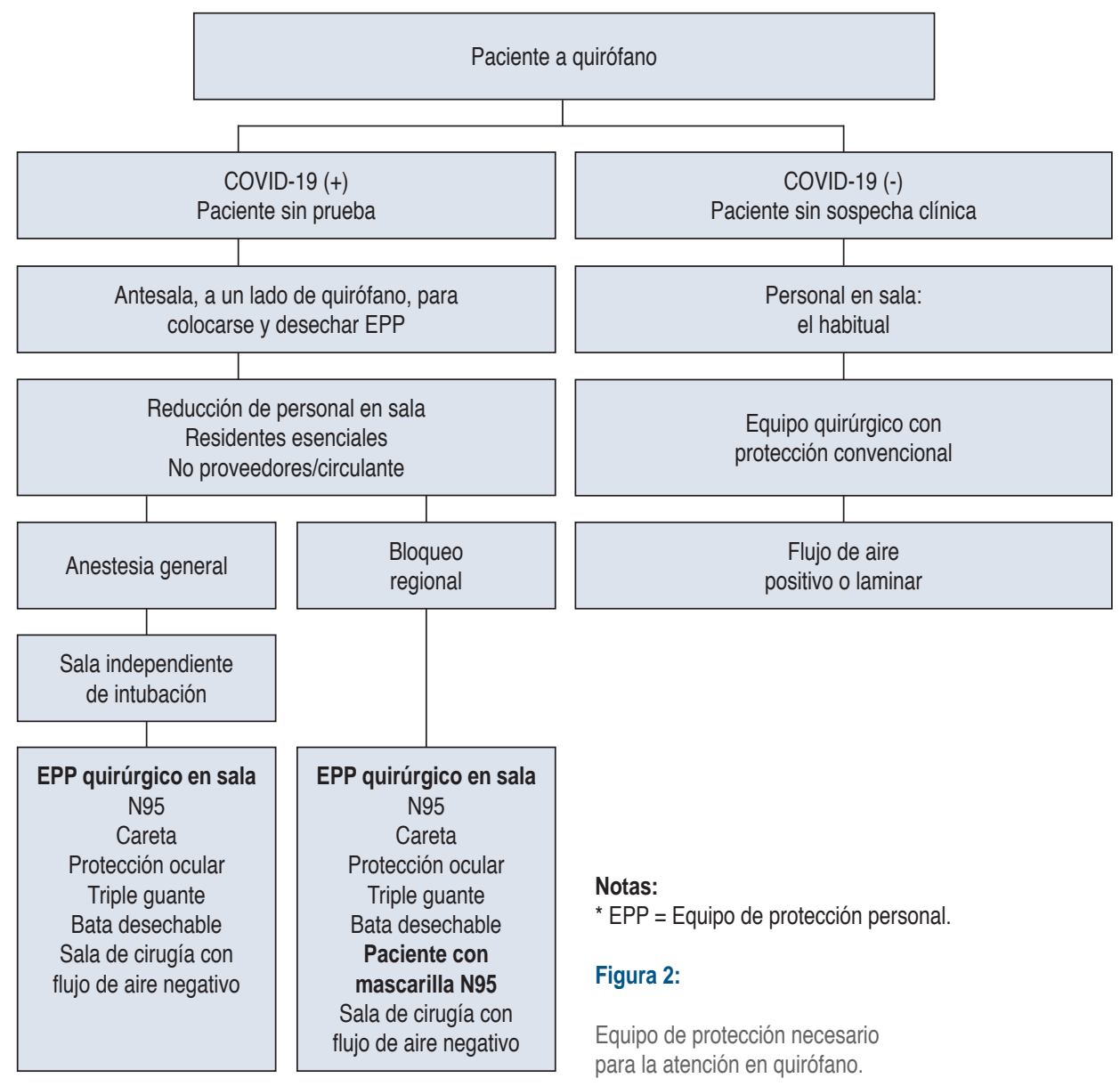

En el área de urgencias, en relación al equipo de protección personal, se sugiere lo recomendado en la Figura 1.

\section{Restringir}

Visitas al hospital por acompañantes, así como a consultorios médicos.

Actualmente la prueba de detección se practica sólo en pacientes que van a ser hospitalizados por sospecha clínica, pero debería realizarse al equipo médico y de esta manera saber si requieren aislamiento por 14 días. La lección de Italia debe aprenderse: los médicos no fueron sometidos a pruebas rutinariamente y las muertes del personal médico son ahora el doble que en China. ${ }^{10}$

Los tiempos en consulta se deben reducir al máximo con un espacio mayor entre cada paciente. 
Utilizar discrecionalmente la aplicación de corticoides de larga acción inyectados, ya que utilizarlos durante la pandemia es preocupante, debido a que puede deprimir el sistema inmunológico. Los analgésicos antiinflamatorios no esteroideos (AINES) han sido asociados con formas más severas de COVID-19. La metilprednisolona ha sido ligada a prolongar la liberación del virus en el huésped, por lo que la OMS recomienda no utilizarla en COVID-19, excepto en pacientes asociados con distrés respiratorio. ${ }^{19}$

\section{DISCUSIÓN}

En esta guía se intenta englobar la mayoría de la práctica clínica de la traumatología, dejando al Ortopedista una toma de decisión basada en evidencia para la practica diaria. Tenemos que poner suma atención a cuál es una urgencia y cuál no, así como qué consulta es necesaria y cuál puede esperar, realizando lo mismo con los procedimientos quirúrgicos. La única manera de hacerlo es estadificando la práctica clínica con base en la evidencia actual.

En cuanto al equipo de protección, es indispensable de acuerdo con las especificaciones realizadas en la Figura 1.

\section{CONCLUSIONES}

La literatura actual nos marca lineamientos claros sobre nuestro actuar ante este tipo de pandemia para evitar más contagios y como salvaguardo legal, aunque su nivel de evidencia es bajo y se ignoran los problemas o costos que esto pudiera generar en el futuro. Se debe de salvaguardar la vida sobre la función. Debemos evitar cometer errores que en otros países se hicieron al menospreciar este brote de COVID-19.

No estabilicemos sólo fracturas, ayudemos a estabilizar pandemias.

\section{BIBLIOGRAFÍA}

1. Zhu N, Zhang D, Wang W, Li X, Yang B, Song J, et al. A Novel Coronavirus from Pa-tients with Pneumonia in China, 2019. New England Journal of Medicine. 2020;382(8):727-33. https://dx.doi.org/10.1056/ NEJMoa2001017

2. World Health Organization. Coronavirus (COVID-19) events as they happen. Who. 2020.

3. https://www.gob.mx/salud/prensa/nuevo-coronavirus-en-el-mundo-covid-19-comunicado-tecnicodiario-238319

4. Secretaría de Salud. SALUD México en twitter: comunicado técnico diario: nuevo coronavirus en el mundo (COVID-19). 2020; 18-19. Available from: https://twitter.com/SSalud_mx/ status/1243357520980107264.

5. Tuček M, Bartoníček J, Novotný P, Voldřich M. Bilateral scapular fractures in adults. Int Orthop. 2013; 37 (4): 659-665.

6. Rothan HA, Byrareddy SN. The epidemiology and pathogenesis of coronavirus disease (COVID-19) outbreak. J Autoimmun. 2020; 109: 102433.

7. Clinical guide for the management of trauma and orthopaedic patients during the coronavirus pandemic. 2020; 1-8.

8. Chang LZ, Wang W, Murphy D, Po Hui JH. Novel coronavirus and orthopaedic surgery. J Bone Joint Surg Am. 2020; 1 : 1.

9. Rodrigues-Pinto R, Sousa R. The orthopaedic forum preparing to perform trauma and orthopaedic surgery on patients with COVID-19. The Journal of Bone and Joint Surgery. 2020; 102(11): 946-950. 
10. Guo X, Wang J, Hu D, Wu L. The orthopaedic forum survey of COVID-19 disease among orthopaedic surgeons in Wuhan, people's republic of China. The Journal of Bone \& Joint Surgery. 2020; 1-15.

11. Disease C. COVID-19: recommendations for management of elective surgical procedures. Am Coll Surg. 2020; 2.

12. Disease B, Work S, Dcis E, Dcis E, Disease N, Work S, et al. COVID-19: elective case triage guidelines for surgical care. 2020; 2020.

13. American College of Surgeons. COVID 19: Considerations for Optimum Surgeon Protection Before, During, and After Operation. 2020; Available from: https://www.facs.org/covid-19/ppe.

14. Colquhoun HL, Levac D, Brien KKO, Straus S, Tricco AC, Perrier L, et al. Scoping reviews: time for clarity in definition, methods, and reporting. J Clin Epidemiol [Internet]. 2014; 67 (12): 1291-1294. Available from: http://dx.doi.org/10.1016/j.jclinepi.2014.03.013.

15. Toro G, Ojeda-thies C, Calabrò G, Toro G, Moretti A, Guerra GM, et al. Management of atypical femoral fracture : a scoping review and comprehensive algorithm. BMC Musculoskelet Disord [Internet]. 2016; 1-13. Available from: http://dx.doi.org/10.1186/s12891-016-1086-8.

16. Stinner DJ, Lebrun C, Hsu JR, Jahangir AA, Mir HR. The Orthopaedic Trauma Service and COVID-19Practice Considerations to Optimize Outcomes and Limit Exposure. J Orthop Trauma. 2020.

17. Joseph B, Hadeed G, Sadoun M, Rhee PM, Weinstein RS. Video consultation for trauma and emergency surgical patients. Crit Care Nurs Q. 2012; 35 (4): 341-345.

18. Barsom EZ, Jansen M, Tanis PJ, van de Ven AWH, Blussé van Oud-Alblas M, Buskens CJ, et al. Video consultation during follow up care: effect on quality of care and patient- and provider attitude in patients with colorectal cancer. Surg Endosc [Internet]. 2020; Available from: https://doi.org/10.1007/ s00464-020-07499-3.

19. FitzGerald GA. Misguided drug advice for COVID-19. Science. 2020; 367 (6485): 1434.

\section{Código ORCID:}

